\title{
Literature Review of Fault Location Research for HVDC Transmission Lines
}

\author{
Wang-Qing Mao ${ }^{1, a, ~}{ }^{,}$, Qi Yang ${ }^{2, ~ b}$, Shuang Zhang ${ }^{3}$, Feng $\mathrm{Gao}^{3}$, Yun-ting Song ${ }^{2, c}$ \\ and Ming-Yu Yang ${ }^{1, d}$ \\ ${ }^{1}$ School of Electrical and Electronic Engineering, North China Electric Power University, Baoding \\ 071003, China \\ ${ }^{2}$ China Electric Power Research Institute, Bejing 100192, China \\ ${ }^{3}$ Ningxia Electric Power Research Institute, Yinchuan 750002, Ningxia Hui Autonomous Region, \\ China \\ amaowangqing@163.com, byangqi@epri.sgcc.com. \\ cn, 'syt@epri.sgcc.com.cn, dyangmy1026@hotmail.com \\ *Corresponding author
}

Keywords: HVDC transmission line, Fault location, Traveling wave method, Fault analysis method, Wavelet transform, Prony.

\begin{abstract}
The HVDC line fault location methods at home and abroad are surveyed in this paper. Firstly, the disadvantage of the existing fault location techniques of HVDC transmission line is analyzed, and it points out that the engineering applications of fault location based on traveling wave principle is most widely used. The fault location principle is relatively simple, but requiring high sampling rate, and its tolerance ability of high resistance is not sufficient. Using the fault location dividing method of AC transmission line for reference, the fault location principle of HVDC transmission line is classified and studied. The HVDC transmission line fault location methods are divided into traveling wave method and fault analysis method. Furthermore, the advantages and disadvantages of each fault location principles of HVDC transmission line are reviewed respectively and it points out that fault analysis method has broad application prospects. Finally, several important problems which need to be further studied are proposed.
\end{abstract}

\section{Introduction}

HVDC transmission technology because of its transportation capacity, small line losses, Non synchronous interconnected power grid, flexible control and other advantages, has been more and more applied in our transmission system.HVDC has been widely used in the "electricity transmitted from west to east", "electricity transmitted from north to sourth" project and it plays an important role in national power grid interconnection ${ }^{[1-9]}$.

DC transmission lines are often thousands of kilometers away, while the DC power lines is precisely the highest failure rate components DC system, operating data also shows that the reliability index of HVDC is on the low side ${ }^{[10]}$. With the development of relay protection technology, fault clearing time of DC line will be shortened, that brings trouble to find the fault point. An accurate and reliable fault location method can reduce line inspection workload, accelerate the speed of crash recovery, reduce the power loss, and guarantee the safe operation of the power system. It has important significance to improve the economy and reliability of the system. DC transmission line fault location technology need to be developed and researched more.

\section{The Research Status of DC Transmission Line}

Fault location is divided into traveling wave method and fault analysis method ${ }^{[1]}$.The DC and AC transmission line are essentially the same in physical, but their energy is concentrated on different frequency bands. This makes parts of fault locating principles of AC line can also be used for DC 
transmission line. At present, the operation of the DC transmission line fault location device adopts the principle of traveling wave only. In fact, the DC line fault location principle can also construct multiple methods, in order to increase the reliability of fault location.

\section{Traveling Wave Method}

Traveling wave method is first used in the AC line.This method makes use of the traveling wave principle, detects the time point that traveling wave head or reflected wave head arriving at the measurement equipment, and then works out the fault location combined with wave velocity.

The principle of fault location based on traveling wave is relatively single. The emphases and difficulties of traveling wave fault location is how to accurately detect the traveling wave head. The traveling wave method can be divided into A type, B type, $\mathrm{C}$ type, $\mathrm{D}$ type, $\mathrm{E}$ type, $\mathrm{F}$ type, among them, the A, C, E, F type need single side data, while the B, D type need double sides data. Type A and type $\mathrm{D}$ are widely used at present.

Type A Traveling Wave Method . Type A method use single end data. Substation at one line end records two arriving times, one is the time that initial fault point's traveling wave get to bus, the other one is the time that traveling wave reflected back to the fault point then reflected back to substation.The distance from the fault point to the substation can be calculated using the time difference and wave velocity to achieve fault location.The hardware requirements of this fault location method are relatively low, only one measurement device installed at one end of the line, communication between two buses is not required.

However, the strength of the wave head get greatly weakened after multiple reflections and refractions at bus point and fault point, which bringing difficulties to wave head detection, especially in many applications. Therefore, this method has not been widely used in engineering practice.

Type D Traveling Wave Method . D-type fault location measurement equipment uses double ends method. It uses the arrival time difference between initial fault wave getting to two ends of the line. D-type device requires the clock at both ends to maintain accurate synchronization which needs the help of global positioning system (GPS). Compared with the single end method,the device cost is higher, but the measurement is more simple and feasible.

At present, the DC line protection and fault location both adopt traveling wave method. As early as in 1985, literature [14] proposed a method using single-ended electrical quantities, the algorithm has high accuracy if opposite end reflection wave and fault point reflection wave can be easily distinguished. Literature [15] gives a traveling wave fault location method using GPS and double-ends electrical quantities, the method has high reliability in the case that both sides of the system are able to start.Literature [16-18] proposed a comprehensive fault location method, it is a combination of single-ended traveling wave location method and double-ends location method, it aims at improving the reliability of the fault location.

\section{Fault Analysis Method}

Fault analysis method is based on the system parameters and the measured voltage, current, the fault distance can be worked out after analysis and calculation.Fault analysis method is simple and feasible, can make use of the existing fault recorder device to achieve the purpose of location. Fault analysis method requires low sampling rate, but higher reliability, and location accuracy can be easily affectted by the accuracy of line parameters. The accuracy of fault analysis method is poor relative to the traveling wave method. Fault analysis method can be divided into the time domain and frequency domain analysis ${ }^{[11-13]}$ according to the electric quantity type.

Fault Analysis Method in Time Domain. In comparison, the method based on distributed parameter model in time domain has obvious advantage over other fault analysis methods when applied on DC transmission line. Full process data from a transient state to steady state can all be used to locate the fault in this method. It requires shorter data window. In addition, protective relay and circuit breaker act more and more quickly, the fault data window is more and more short, this trend 
has also forced fault location technique towards the direction of distributed parameter model and time domain.

Literature [19-20] proposed a fault method based on distributed parameter model and time domain, this method uses circuit and voltage variable of both ends of line.The method is performed in time domain, without filtering, can use any piece of data from transient state to steady state.Literature [21] presented a single terminal fault location principle of DC line in time domain, its basic idea is to use fault current provided by the rectifier, calculate of voltage and current distribution along in the distributed parameter model, and realize the fault location according to boundary conditions. The literature [22] is based on literature [21], a genetic algorithm was used in order to improve the positioning accuracy in case that the transmission line parameters is not accurate.

Fault Analysis Method in Frequency Domain.Literature [23-24] proposed a new method of single end based on the extraction of natural frequencies. Wave in an ideal transmission line, when the wave is fully reflected at both ends, represents an infinite number of harmonics of a specific frequency in frequency domain, called the natural frequency of the traveling wave. The natural frequency is determined by the total length of the line and the wave velocity. After a fault, due to the occurrence of reflection and refraction at fault point, the natural frequency generates. The method uses the frequency and the corresponding velocity to calculate fault distance.The natural frequency is from distribution parameter characteristics and traveling wave reflection in essence.The natural frequency of HVDC transmission line is much stronger compared with that of the AC line. Since the method based on the natural frequency do not need to identify wave head and using only random transient fault data to extract natural frequency, it can be more accurate and reliable compared to method needing identification of traveling wave head.

\section{Summary}

This paper makes a comprehensive summary of the DC transmission line fault location principle. Based on the summary of research and practice at home and abroad, fault location methods are classified, this paper points out that the fault location applied on DC transmission line at present exists problems such as a single principle, high sampling rate, not high reliability.

DC transmission line and AC transmission lines have no essential difference, but different concentration on energy band. In theory, part of AC line's fault location principle is also applicable to the DC line.The fault transient wave of DC system contains a lot of characteristic frequency, fault location research based on the characteristic frequency can be studied. Time domain fault location method based on differential equation, the principle is not affected by the non periodic component and harmonic effects. Make full use of the fault characteristics of DC transmission line, a variety of fault location principle can be constructed, so as to improve the accuracy and reliability of DC line fault location .

\section{References}

[1] Yaozhong Ge: The new relay protection and fault location principles and techniques ( Xi'an Jiaotong University Press, Xi'an 2007,in Chinese).

[2] Research group of HVDC from Power Department of Zhejiang University: DC transmission, (Hydraulic and Electric Power Press, Beijing,1985,in Chinese).

[3] Wanjun Zhao: High voltage DC transmission engineering technology ( Chinese Electric Power Press, Beijing, 2004, in Chinese).

[4] Zheng Xu: Dynamic behavior analysis of DC and AC power system ( Mechanical Industry Press, Beijing, 2004, in Chinese).

[5] J.G. Holm: Transactions of the American Institute of Electrical Engineers, Vol. 72(1953), p. 1114-1120. 
[6] B.O.N. Hansson: Transactions of the American Institute of Electrical Engineers, Vol. 73 (1954), p. 599-605.

[7] J.G. Holm: Transactions of the American Institute of Electrical Engineers, Vol. 76(1957), p. 849-855.

[8] J.F. Perrin: Journal of the Institution of Electrical Engineers, Vol. 7(1961), p. 559.

[9] W. Long, S. Nilsson: IEEE Power and Energy Magazine, Vol. 5(2007), p. 22-31.

[10] Haifeng Liu, Xu Zheng , Licheng Jin: High Voltage Apparatus, Vol. 38(2002), p. 1-4,in Chinese.

[11] Chaoran Ma: Prospect and development of transmission line fault location using travelling wave, Relay, Vol. 35(2007), p. 11-15,in Chinese.

[12] Qiang Li: Power System Protection and Control, Vol. 38(2009), p. 192-197,in Chinese.

[13] Junhong Guo, Weipu Tan, Yihan Yang: Relay, Vol. 34(2006), pp. 76-81, in Chinese.

[14] Ando Masaoki, E.O.Schweitzer, R.A. Baker: IEEE Transactions on Power Apparatus and Systems, Vol. 104(1985), p. 3531-3537.

[15] M.B. Dewe, S. Sankar, J.Arrillaga: IEEE Transactions on Power Delivery, Vol. 8(1993), p.1295-1302.

[16] Yongchang Zhai, Haifeng Zhang: IET Conference Publications, 2006, p.490-496, in Chinese.

[17] Ping Chen, Bingyin Xu, Jing Li: 2006 International Conference on Power System Technology (2006 POWERCON), 2006 ,p.1-4.

[18] JingXie, Ping Chen: Journal of Shandong University of Technology(Sci\&Tech), Vol. 20(2006), p.47-50,in chinese.

[19] Guobing Song, Desheng Zhou, Zaibin Jiao: Automation of Electric Power Systems, Vol. 31(2007), p.57-61,in Chinese.

[20] Guobing Song, Jiale Suonan: Proceedings of the CSEE, Vol. 24(2004), p.24-29, in Chinese.

[21] Shuping Gao, Jiale Suonan, Guobing Song: Proceedings of the CSEE,Vol. 13(2010),p. 75-80,in Chinese.

[22] Hongbo Li: Research on theory of fault location and software development for HVDC lines, Tianjin University, Tianjin, 2009,in Chinese.

[23] Yonglin Wu, Zhengyou He, Qingquan Qian: Proceedings of the CSEE, Vol. 28(2008), p.69-75, in Chinese.

[24] Yonglin Wu, Zhengyou He, Qingquan Qian: Proceedings of the CSEE, Vol. 25(2008), p.99-104,in Chinese. 